\title{
Multi-Site Split Learning for Privacy-Preserving Medical Systems under Data Imbalance Constraints: A Feasibility Study with COVID-19, X- Ray, and Cholesterol Dataset
}

\author{
Yoo Jeong $\mathrm{Ha}$ \\ Korea University \\ Gusang Lee \\ Korea University \\ Minjae Yoo \\ Korea University \\ Soyi Jung \\ Hallym University \\ Seehwan Yoo \\ Dankook University \\ Joongheon Kim ( $\sim$ joongheon@korea.ac.kr) \\ Korea University
}

\section{Research Article}

Keywords: COVID-19, data sharing technology, X-ray bone scans

Posted Date: September 21st, 2021

DOI: https://doi.org/10.21203/rs.3.rs-888423/v1

License: (c) (i) This work is licensed under a Creative Commons Attribution 4.0 International License.

Read Full License

Version of Record: A version of this preprint was published at Scientific Reports on January 27th, 2022. See the published version at https://doi.org/10.1038/s41598-022-05615-y. 


\title{
Multi-Site Split Learning for Privacy-Preserving Medical Systems under Data Imbalance Constraints: A Feasibility Study with COVID-19, X-Ray, and Cholesterol Dataset
}

\author{
Yoo Jeong Ha ${ }^{1}$, Gusang Lee ${ }^{1}$, Minjae Yoo ${ }^{1}$, Soyi Jung ${ }^{2, *}$, Seehwan Yoo ${ }^{3, * *}$, and \\ Joongheon $\mathrm{Kim}^{1, * * *}$
}

${ }^{1}$ Korea University, School of Electrical Engineering, Seoul, 02841, Republic of Korea

${ }^{2}$ Hallym University, School of Software, Chuncheon, 24252, Republic of Korea

${ }^{3}$ Dankook University, Department of Mobile Systems Engineering, Yongin, 16890, Republic of Korea

*sjung@hallym.ac.kr

**seehwan.yoo@dankook.ac.kr

****joongheon@korea.ac.kr

\begin{abstract}
It seems as though progressively more people are in the race to upload content, data, and information online; and hospitals haven't neglected this trend either. Hospitals are now at the forefront for multi-site medical data sharing to provide groundbreaking advancements in the way health records are shared and patients are diagnosed. Sharing of medical data is essential in modern medical research. Yet, as with all data sharing technology, the challenge is to balance improved treatment with protecting patient's personal information. This paper provides a novel split learning algorithm coined the term, "multi-site split learning", which enables a secure transfer of medical data between multiple hospitals without fear of exposing personal data contained in patient records. It also explores the effects of varying the number of end-systems and the ratio of data-imbalance on the deep learning performance. A guideline for the most optimal configuration of split learning that ensures privacy of patient data whilst achieving performance is empirically given. We argue the benefits of our multi-site split learning algorithm, especially regarding the privacy preserving factor, using CT scans of COVID-19 patients, X-ray bone scans, and cholesterol level medical data.
\end{abstract}

\section{Introduction}

The world was met with a demise when the lethal Coronavirus Disease 2019 (COVID-19) began taking away the lives of loved ones. COVID-19 has infected 221,648,869 and killed 4,582,338 people worldwide as of September 8th, 2021 ${ }^{1}$. Since both case numbers and deaths linked to COVID-19 continue to rise as new and more aggressive variants of the contagion reach the population, the world is shifting further away from the end of the COVID-19 pandemic. This ongoing global pandemic has divided the world apart at a time where mankind should unite to fight this fatal disease together. At the heart of this step to amalgamate the deeply detached, is our proposed "multi-site split learning" algorithm. This paper aims to furnish a secure learning process where hospitals all over the globe can share their findings to create a deep learning model without revealing any sensitive patient information. This new technique allows hospitals to share their data without sending raw medical images to external associations. Our privacy-enhancing technology provides experimental results using real-world data, with only an encrypted image being divulged to an external server.

In split learning, the learning process is, as the word suggests, literally split or separated into two parts: the end-systems and the server. Here, the end-systems refer to the multiple hospitals that possess original medical data to be trained in the deep learning model. The server is where the actual learning of the deep neural network occurs. Multiple hospitals are connected to one server; all the hospitals connected to the server collaborate to train the very deep neural network that is placed in the server without exposing their raw data to an external network. A meticulously devised framework of our proposed algorithm is shown in Figure 1. Every individual hospital only runs the deep neural network up to the first hidden layer. After passing through the first hidden layer, the end-systems send only their feature maps to the server. Since only the encrypted feature map is transferred to the external network, and not the original medical data, the privacy of confidential patient information is preserved. It is coined "multi-site split learning" since multiple hospitals (multi-site) are geometrically spaced apart from each other and the deep neural network is divided among them. 
As more citizens are hospitalized due to the COVID-19 virus, a precise identification tool in the hospital to help accelerate the treatment process is imperative. This powerful tool can be utilized with various types of data such as image data or numerical data. With the appropriate kind of deep learning model selected, our algorithm is applicable to accompany a wide variety of data types. It has been reported that COVID-19 causes severe acute respiratory syndrome coronavirus 2 (SARA-CoV-2). With the deployment of our novel technique, a precise classification of pneumonia caused by the COVID-19 virus can be made. This dataset is utilized in such a timely manner for this pandemic; this technological assistance can serve as a means to slowing down the number of patients getting infected with SARA-CoV-2 by devising initial treatment plans. Also, we use MUsculoskeletal RAdiographs (MURA) datasets, a collection of X-ray scans from seven body parts, to classify whether a bone is fractured or not. According to ${ }^{2}$, the musculoskeletal condition affects one in two American adults, which accounts for far more than that of cardiovascular and chronic respiratory diseases combined. Hence, this dataset is used to validate our model and aid in treating this common illness. Since our algorithm can be applied to different data types, we also use numerical data-cholesterol levels-to predict the level of harmful cholesterol using other patient information. We use three medical datasets to corroborate our claim on the benefits of our multi-site split learning algorithm.

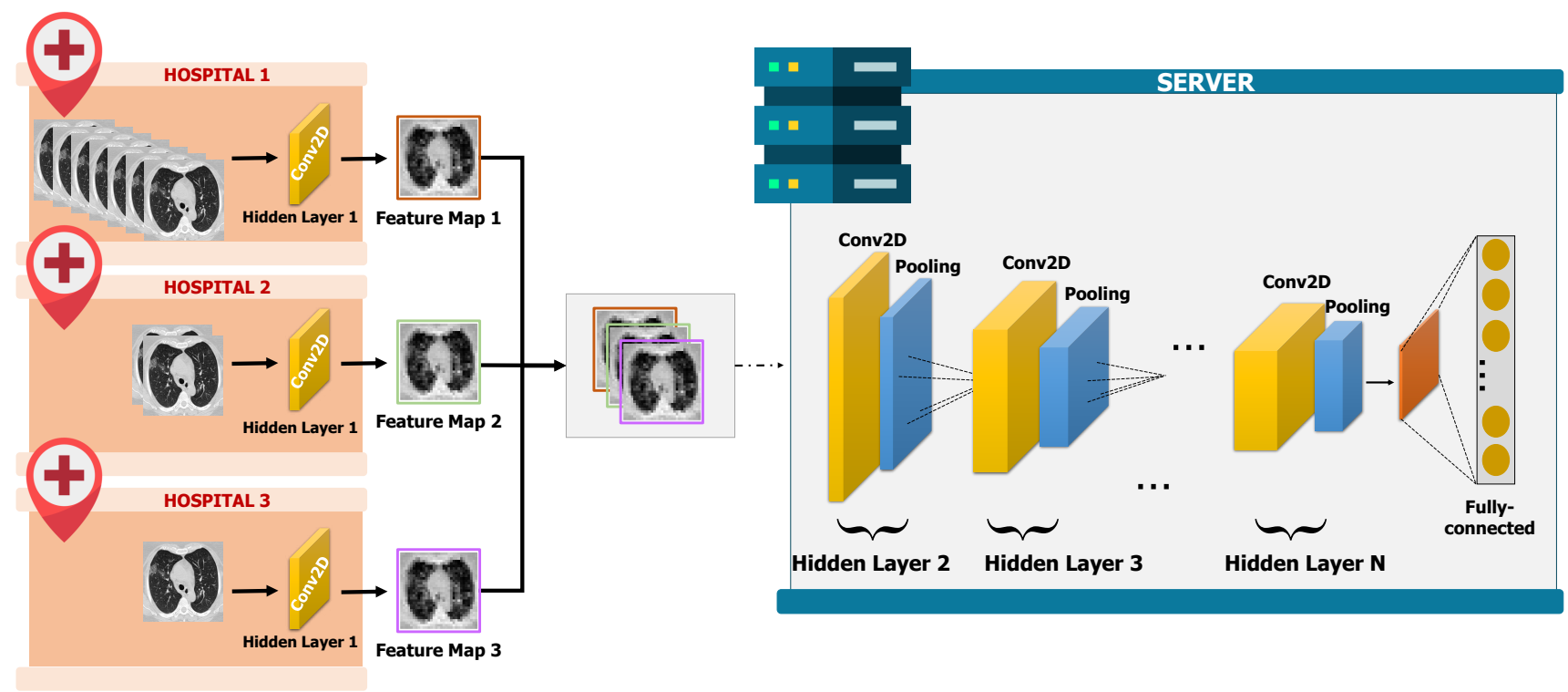

Figure 1. The overall framework of our multi-site split learning algorithm.

\section{Results}

\section{Preserving Privacy of Medical Data}

The anticipation of today's rapid epochal shift into the information and technology era extends into the interoperability of medical data with external research partnerships. The sharing of medical information between health care provides is extremely advantageous to both the patient and physicians as well. The global health crisis is shining a harsh spotlight on healthcare's biggest loopholes; the benefits of data sharing come hand-in-hand with the risks of unscrupulous hackers getting access to personal information. The ominous presence of hackers in cyberspace creates a fear of leaks of sensitive patient information wherever it gets transferred.

Therefore, one of the paramount benefits of our proposed multi-site split learning algorithm is the protection of the original training data. Since the deep neural networks are, as the word suggests, literally split between the multiple end-systems and the server. Each of the participating hospitals, or end-systems, only has one hidden layer, and the rest of the hidden layers are located in the server. After running the deep neural network up to the first hidden layer, the feature maps from all the active hospitals are sent to the server, where they are concatenated. This concatenated feature map becomes the input to the server's deep neural network. The feature map is the sole information that is exposed to a third-party affiliation; the original medical data does not get shared between the participating hospitals. 


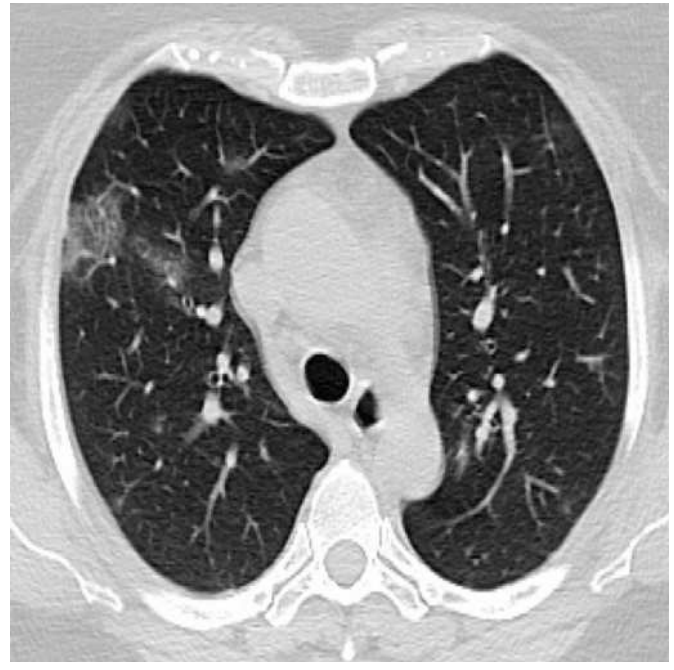

(a)

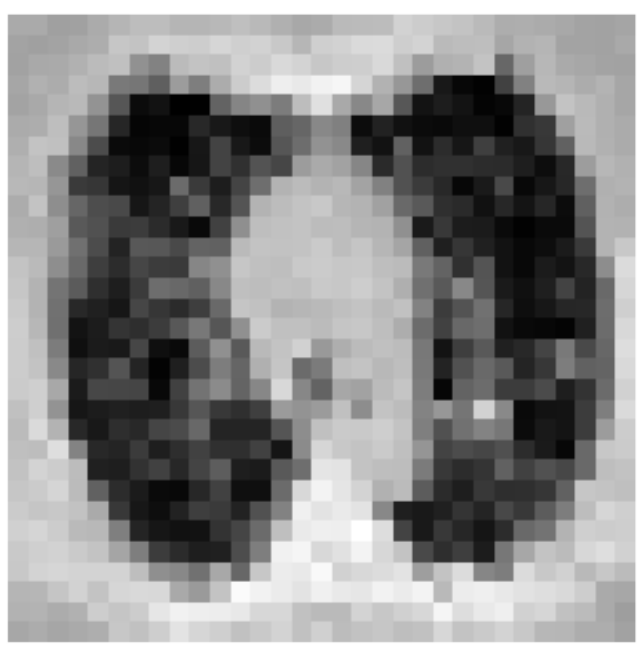

(b)

Figure 2. Ensuring privacy of original medical data. Image (a) is the original image of the COVID-19 CT scan. Image (b) shows the highly distorted image after passing through one hidden layer at the hospitals.

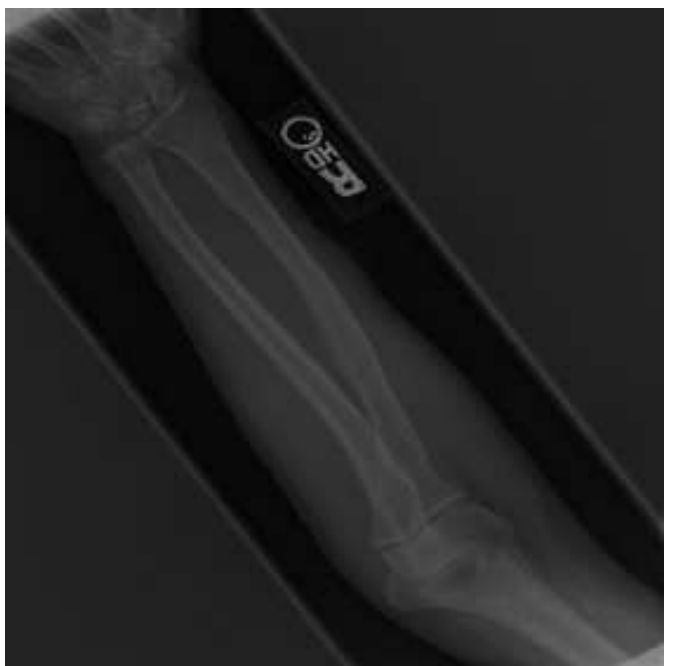

(a)

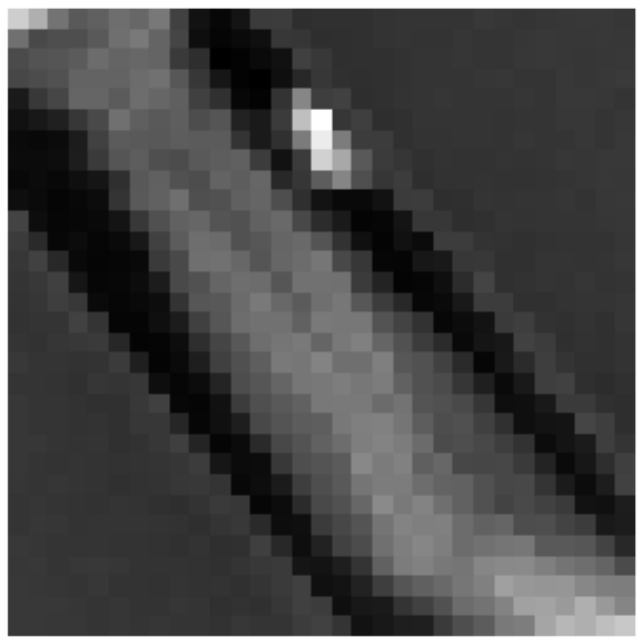

(b)

Figure 3. Ensuring privacy of original medical data. Image (a) is the original image of the MURA bone X-ray scans. Image (b) shows the highly distorted image after passing through one hidden layer at the hospitals.

The extent to which the raw medical data is protected is shown in the following Figures. Figure 2 shows the degree to which the original COVID-19 CT scan is distorted after passing through one hidden layer of each hospital. Figure 2 (a) is the original CT scan of a COVID-19 patient. Figure 2 (b) is the highly deformed image of image (a) after it undergoes the first hidden layer. Each hospital will produce an image like Figure 2 (b). All of these feature maps are concatenated and communicated to the server where it then trains the deep neural network.

Similarly, the protection of patient records applies to MURA bone X-ray scans as well. Each hospital possesses raw images of MURA bone X-ray scans. These hospitals run the deep neural network only up to the first hidden layer using their own raw medial data, which is displayed in Figure 3 (a). The output of the first hidden layer, which is depicted in Figure 3 (b), is collected from each of the end-systems. The second image is the only information that is revealed to the server. Hence, the original medical image is protected from any and all external threats. Furthermore, as evidently demonstrated in the comparison between Figure 3 (a) and (b), even if hackers do obtain possession of the feature maps when transferring to the server, it will be very difficult to trace it back to the original image. Once again, protecting the privacy of patient information.

The same principle applies to numerical data. Just as the combination of the Conv2D and MaxPooling2D layer, in the 
server's end shown in Figure 1, alters the original data to a state where it is unrecognizable, the original cholesterol data are also deformed to protect the raw patient records in the same manner.

\section{Effect of Varying Number of End-systems}

It is conspicuous that data exchange in regards to medical information opens doors for meaningful research. Our split learning model is a cohesive ecosystem that seamlessly works to achieve one goal, which is to provide quality care for patients. It is a safe and trustworthy technique that entities outside of a particular hospital, such as pharmaceutical companies, clinical and molecular research centers, can leverage their expertise to avoid medication errors. The pandemic has instilled the need for patient-centric data sharing across clinical institutions to help understand the integral effect of this unprecedented virus. Extending further from just the pandemic, sharing medical data among other hospitals can reduce doctor visits or hospital admissions.

In this experiment, we examine the effect of differing the number of end-systems on classification performance. Our algorithm is set up to emulate a real case scenario where multiple hospitals come together to share their sources and collaborate to train a deep neural network. As discussed in the introduction, more hospitals are wanting to co-operate with others in medical research and data-sharing. We test the outcomes according to the number of participating hospitals, accurately presenting when the split learning can achieve better performance by collaboration.

Hence, the experiment is devised with a varying number of end-systems, i.e., hospitals. Each of the hospitals transfers only the feature maps up to the centralized server. In our split learning design, the deep neural network is divided in the following way. Each hospital runs the neural network up to the first hidden layer using its own unique data. Then the output of that first hidden layer, which is the feature map, is sent to the server. The server takes the feature map as input, and continues training with the rest of the hidden layers. Hence, the end-systems only have one hidden layer and the server is comprised of the rest of the hidden layers, which is where the majority of the computation is performed. This divided deep neural network setup is the same for all the participating hospitals, whether there are 3, 4, or 5 contributing hospitals.

\section{Result on COVID-19}

This experiment classifies the CT scans into two groups: those suffering from SARA-CoV-2 and those healthy without any chest pain. The chest CT scans of COVID-19 patients and non-COVID-19 patients are processed under a convolutional neural network (CNN). Using a loss function of binary crossentropy, an activation function of sigmoid, a batch size of 64, and an input size of $64 \times 64 \times 1$, the CT scan images are classified. The experiment setup is summarized in Table 4 .

In this work, we test the efficacy of increasing the number of cooperating hospitals in a split learning environment. Furthermore, we give an empirical guideline to the optimal number of hospitals for the highest accuracy with the lowest loss. The two major patterns of increasing the number of end-systems only are highlighted below:

- Overfitting can be prevented when there are fewer end-systems, yet it is difficult for performance to improve as the number of end-systems increases.

- Once the number of end-systems reaches five, loss takes longer to converge and stabilize.

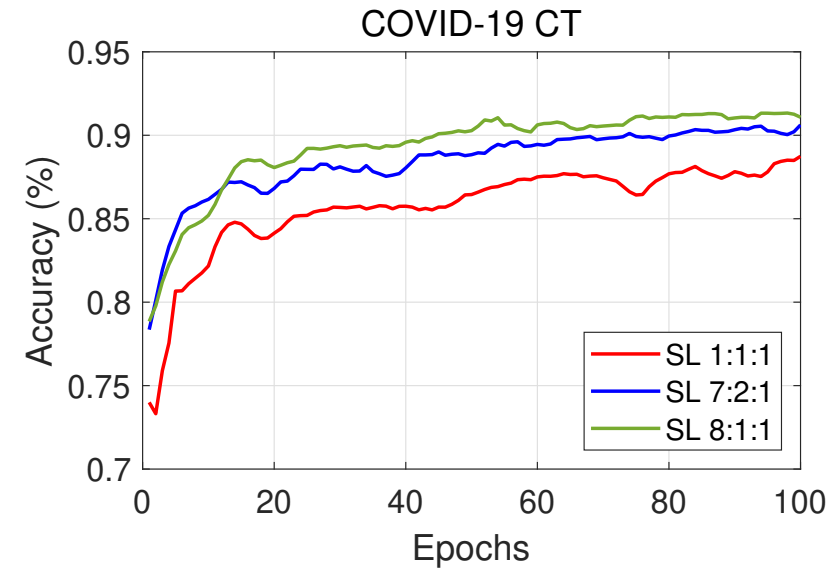

(a) Accuracy with 3 end-systems.

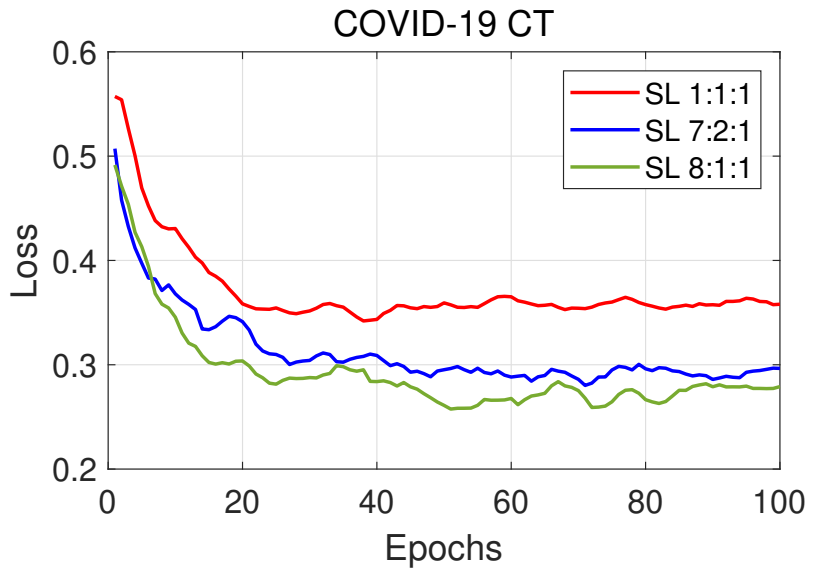

(b) Loss with 3 end-systems.

Figure 4. Performance tested with three end-systems. The graph depicts the classification results with three end-systems having 1:1:1, 7:2:1 and 8:1:1 data-imbalance. 


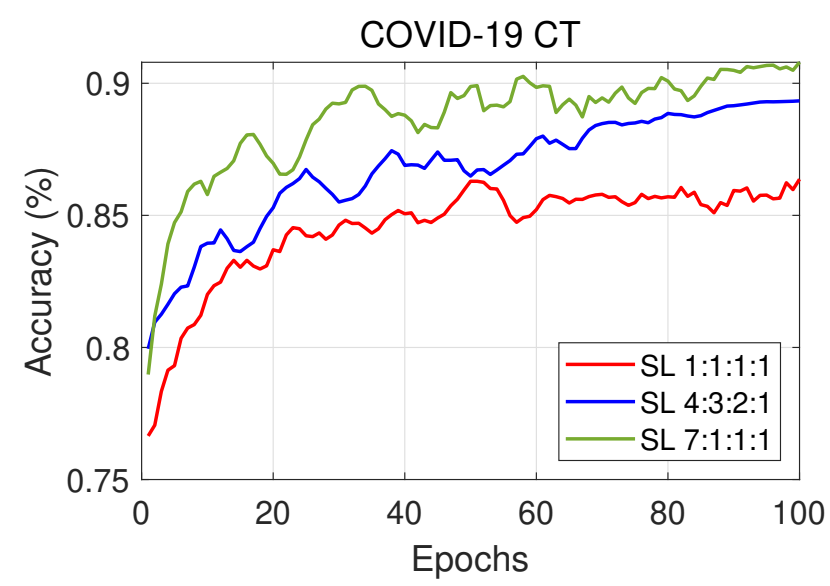

(a) Accuracy with 4 end-systems.

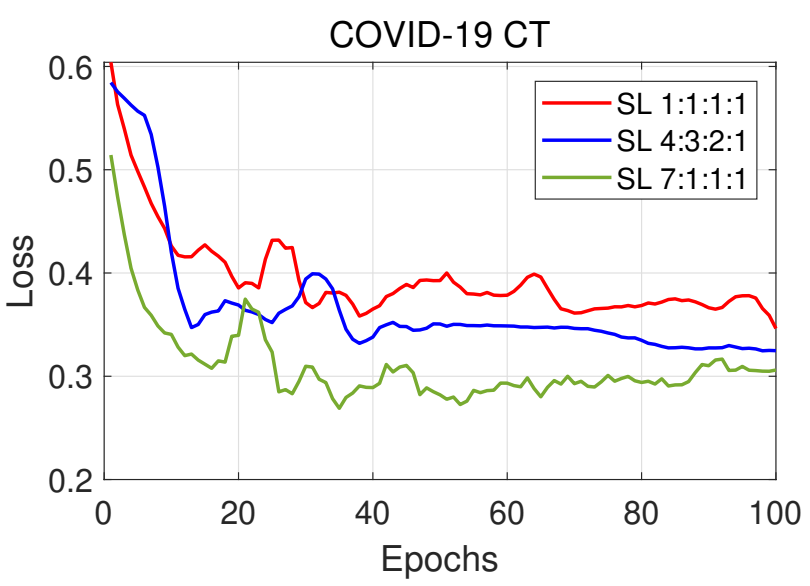

(b) Loss with 4 end-systems.

Figure 5. Performance tested with four end-systems. The graph depicts the classification results with four end-systems having 1:1:1:1, 4:3:2:1 and 7:1:1:1 data-imbalance.

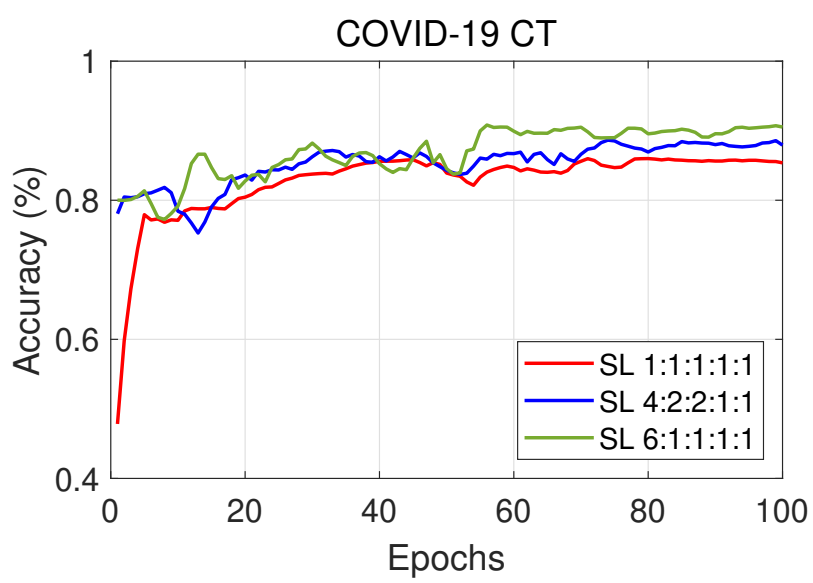

(a) Accuracy with 5 end-systems.

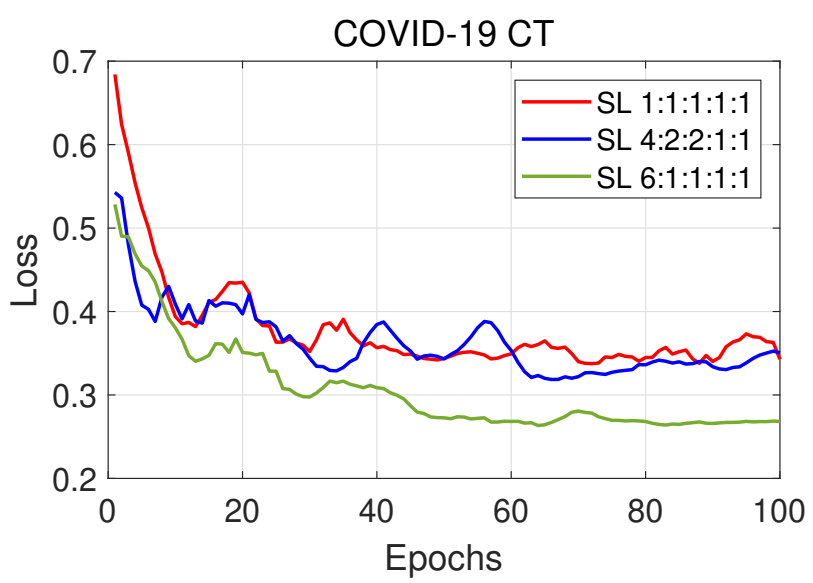

(b) Loss with 5 end-systems.

Figure 6. Performance tested with five end-systems. The graph depicts the classification results with four end-systems having 1:1:1:1:1, 4:2:2:1:1 and 6:1:1:1:1 data-imbalance.

Comparing Figure 4 with Figure 5 and with Figure 6, a visual contrast between the accuracy and loss patterns can be made. Only considering the increasing number of end-systems, and not the split ratios, it is evident that the classification accuracy decreases as more hospitals join split learning. This pattern can be seen when comparing the subsequent data ratio of each end-system with its corresponding ratio. An equal division of the datasets between the corresponding end-systems is a default ratio and will be the acting benchmark for fair comparisons. Otherwise stated, an equally divided 1:1:1 ratio, a 1:1:1:1 ratio, and a 1:1:1:1:1 ratio for when there are 3, 4, and 5 end-systems respectively. This marks the base for a fair comparison rather than looking at the 7:2:1, 4:3:2:1, and 4:2:2:1:1 ratio group to make conclusions on varying the number of end-systems.

The same observation can be made with proof from the loss graphs. It is more meaningful to judge the rate of convergence in the case of loss graphs. In comparing Figure 4 (b) and Figure 5 (b), it is evident that a lower loss value is reached at a smaller amount of epochs for 3 end-systems than that of 4 end-systems. The loss curves for 3 hospitals stabilize quicker and converge at a relatively lower loss value. When one more end-system is added and Figure 6 (b) comes into the picture, the loss value reaches a stable point at a higher level. This validates our claim that the performance levels fall as more hospitals engage in split learning. 


\begin{tabular}{|c|c|c|c|c|c|c|c|c|c|}
\hline Number of end-systems & \multicolumn{3}{|c|}{3} & \multicolumn{3}{c|}{4} & \multicolumn{3}{c|}{5} \\
\hline Split ratio & $1: 1: 1$ & $7: 2: 1$ & $8: 1: 1$ & $1: 1: 1: 1$ & $4: 3: 2: 1$ & $7: 1: 1: 1$ & $1: 1: 1: 1: 1$ & $4: 2: 2: 1: 1$ & $6: 1: 1: 1: 1$ \\
\hline Accuracy(\%) & 88.3 & 90.1 & 91.4 & 85.7 & 89.3 & 90.8 & 85.6 & 88.8 & 90.5 \\
\hline
\end{tabular}

Table 1. Classification accuracy using COVID-19 CT scan.

Furthermore, Table 1 shows the exact accuracy level at which all the end-systems converge. Having these values displayed side-by-side, the highest accuracy level of $91.4 \%$ is achieved by our split learning algorithm experimented with three endsystems.

\section{Result on MURA}

The same test is conducted using MURA datasets and the experiment setup is summarized in Table 4. Here, we want to observe the effect of increasing the number of end-systems in split learning has on the classification accuracy using a different medical dataset. Since this test is conducted with MURA, it gets X-ray scans of each of the seven body parts as inputs:fingers, elbows, forearms, hands, humerus, shoulders, or writs. The X-ray scans go through one hidden layer at the hospital and the output feature map is sent to the server. Once the feature map is received at the server's end, the concrete CNN procedure proceeds. The server will determine whether or not the patient is suffering from musculoskeletal disorders from the feature maps of the $\mathrm{X}$-ray scans.

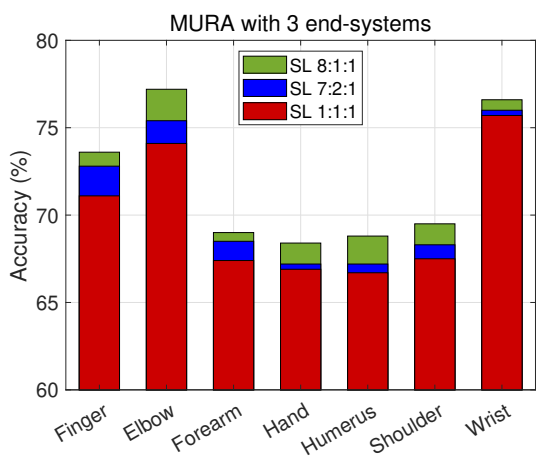

(a) Accuracy with 3 end-systems.

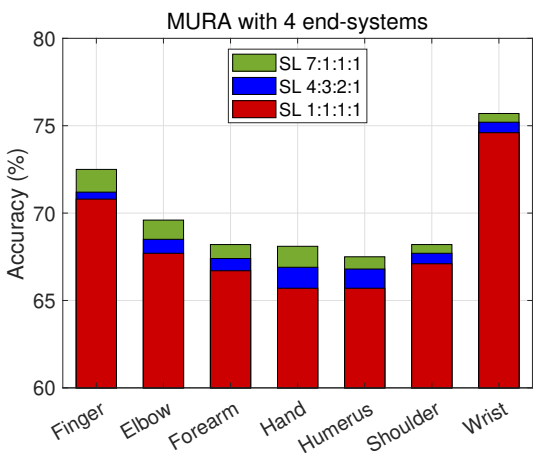

(b) Accuracy with 4 end-systems.

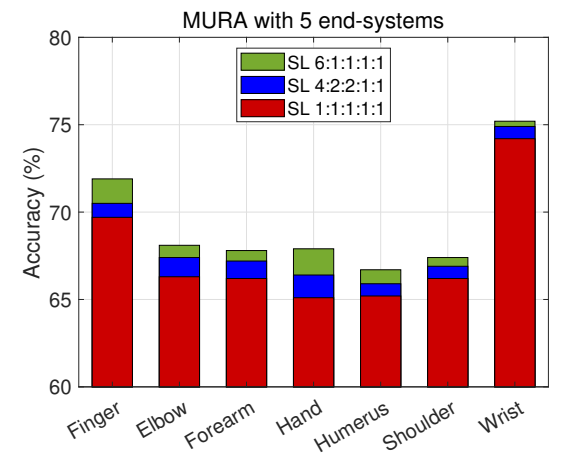

(c) Accuracy with 5 end-systems.

Figure 7. Accuracy of the seven body parts using MURA dataset for (a) 3, (b) 4, and (c) five end-systems.

\begin{tabular}{ll|ccc|ccc|ccc}
\hline & \multicolumn{9}{c}{ MURA Accuracy(\%) } \\
\cline { 2 - 11 } & $\begin{array}{l}\text { Number of } \\
\text { end-systems }\end{array}$ & \multicolumn{2}{c}{3} & & \multicolumn{3}{c|}{4} & \multicolumn{3}{c}{5} \\
\cline { 2 - 10 } & Split ratio & $1: 1: 1$ & $7: 2: 1$ & $8: 1: 1$ & $1: 1: 1: 1$ & $4: 3: 2: 1$ & $7: 1: 1: 1$ & $1: 1: 1: 1: 1$ & $4: 2: 2: 1: 1$ & $6: 1: 1: 1: 1$ \\
\hline \multirow{4}{*}{ MURA } & Finger & 71.1 & 72.8 & 73.6 & 70.8 & 71.2 & 72.5 & 69.7 & 70.5 & 71.9 \\
body & Flbow & 74.1 & 75.4 & 77.2 & 72.9 & 73.2 & 75.2 & 72.0 & 72.6 & 73.7 \\
parts & Hand & 67.4 & 68.5 & 69.0 & 66.7 & 67.4 & 68.2 & 66.2 & 67.2 & 67.8 \\
& Humerus & 66.9 & 67.2 & 68.4 & 65.7 & 66.9 & 68.1 & 65.2 & 66.4 & 67.9 \\
& Shoulder & 67.5 & 67.2 & 68.8 & 65.7 & 66.8 & 67.5 & 65.2 & 65.9 & 66.7 \\
& Wrist & 75.7 & 76.0 & 76.5 & 67.1 & 67.7 & 68.2 & 66.2 & 66.9 & 67.4 \\
\hline
\end{tabular}

Table 2. Classification accuracy using MURA body parts.

The results for this experiment are graphed in Figure 7, with the classification accuracy for each body part shown. In comparison between the number of end-systems, we look at the highest accuracy out of the three split ratios. With the finger $\mathrm{X}$-ray images, the performance with 3 hospitals training CNN under equally divided data ratio has a classification accuracy of $71.1 \%$, with 4 it drops to an accuracy of $70.8 \%$, and with 5 end-systems it achieves $96.7 \%$, as seen in Table 2. For the elbow scans, split learning with 3 end-systems reaches up to $74.1 \%$ accuracy, $72.9 \%$ for 4 end-systems, and $72.0 \%$ for 5 hospitals. Analyzing the rest of the body parts, a general trending pattern is observed. When only considering the number of end-systems, it is concluded that 3 end-systems give the best image classification performance and. Therefore, as the number of end-systems 
increases, the classification accuracy level decreases. This result coincides with the analysis made with the COVID-19 chest CT scan data as well.

\section{Result on Cholesterol}

The split learning algorithm is a versatile tool that can train any type of data. With an appropriate model selected, split learning can be used to train image data or even numerical data as well. With the previous two COVID-19 and MURA scans, we saw that image data is processed to give aid in medical treatment plans. In this section, we see the results of split learning using cholesterol data. The experiment is conducted to predict the so-called Low-density lipoprotein cholesterol (LDL-C) level using other patient attributes such as age, sex, height, and weight. This regression model is used to predict the level of LDL-C in the blood and the performance is tested using root mean squared logarithmic error (RMSLE). RMSLE is a standard means to compare the loss value between the original and the predicted data in a regression model.

\begin{tabular}{|c|c|c|c|c|c|c|c|c|c|}
\hline Number of end-systems & \multicolumn{3}{|c|}{3} & \multicolumn{3}{c|}{4} & \multicolumn{3}{c|}{5} \\
\hline Split ratio & $1: 1: 1$ & $7: 2: 1$ & $8: 1: 1$ & $1: 1: 1: 1$ & $4: 3: 2: 1$ & $7: 1: 1: 1$ & $1: 1: 1: 1: 1$ & $4: 2: 2: 1: 1$ & $6: 1: 1: 1: 1$ \\
\hline RMSLE & 0.0613 & 0.0597 & 0.0567 & 0.0651 & 0.0624 & 0.0593 & 0.0674 & 0.0658 & 0.0616 \\
\hline
\end{tabular}

Table 3. The RMSLE loss values for cholesterol data. It shows the loss for each end-system (3, 4, 5 end-systems) with varying data-ratios.

Looking at the results of the experiment tested with varying the number of end-systems in Table 3, a noticeable and repeating pattern can be observed. The table shows the loss value, i.e. the difference between the actual and predicted value, the lower the loss, the better the result. RMSLE records a loss of 0.0613 with 3 end-systems, 0.0651 with 4 end-systems, and 0.0674 loss with 5 end-systems when the data are equally split. Hence, a general trend is noted with the cholesterol data-with an increasing number of end-systems, the performance of split learning falls. This observation is consistent with the trends seen using the two image datasets. Therefore, when the number of end-systems is considered only, the highest prediction performance is seen when 3 hospitals engage in split learning.

\section{Effect of Changing the Data Ratio}

Previously in the above section, this paper explored the impact of varying the number of end-systems, i.e. hospitals, has on the performance of our split learning algorithm. In this section, we will investigate the outcome of changing the data-imbalance split ratio. Data-imbalance is a prevalent issue that occurs in data-sharing. This experiment is strategically calculated to simulate the real-life issue medical sectors face. In reality, one hospital may possess a large number of data on magnetic resonance imaging (MRI). Yet, another hospital may lack data in that department, which makes that hospital inadequate in making an accurate informed medical diagnosis. This is where our proposed split learning comes in handy. When this small hospital becomes a participant in this split learning algorithm, it can seek the benefits of other hospital's data without risk of exposing sensitive patient information. In our study, the data ratio is a representation of the hospital scale-a small split ratio portion is assigned to an end-system that holds a small amount of data, which is symbolic of a small-scale hospital. Similarly, the portion that is a relatively big data split ratio represents a large-scale hospital that holds voluminous amounts of data.

Therefore, to reflect on this reality, the experiment is arranged so that each of the training datasets is divided into different ratios. For example, if 4 hospitals are collaborating to build a deep neural network, we can divide the datasets into a 4:3:2:1 ratio. That is, one hospital is assigned to have $40 \%$ of the data, the other hospital will possess $30 \%$ of the data, another hospital will have $20 \%$ of the data, and the last hospital will have the remaining $10 \%$ of the data. By dividing the datasets into these unique ratios, our study speculates the reality of data-imbalance in the medical sector and delves into exploring the effect of having different amounts of data in split learning.

Referring back to Table 1, a deep analysis on the effect of varying the split ratio is conducted. Three different ratio divisions are considered for each of the separate end-systems. First of all, let's have a look at the split ratio for when 3 hospitals are training a deep neural network. When all 3 end-systems divide the COVID-19 dataset equally, the classification accuracy is $88.3 \%$. As it is rather unlikely that all 3 hospitals share an equal amount of data, the data is split into a 7:2:1 ratio among the 3 end-systems. This gives a classification accuracy for COVID-19 chest CT scans of $90.1 \%$. Then, another variation is given, with a ratio of 8:1:1, which gives $91.4 \%$ performance. For the same reasoning, a split among the datasets is made when 4 hospitals engage in split learning. As seen in Table 1 and Figure 5, with a 1:1:1:1 ratio, the accuracy level reaches 85.7\%. A split ratio of 4:3:2:1 performs $89.3 \%$ and that of 7:1:1:1 reaches a classification accuracy of $90.8 \%$. Similarly, test results for the data split when there are 5 end-systems are shown in Table 1 and Figure 6 . The experimental results show that as the split ratio varies from equal $(1: 1: 1: 1)$ to data-imbalance (4:3:2:1) to extreme data-imbalance ( $7: 1: 1: 1)$, the classification accuracy increases for all 3, 4, and 5 end-systems. 
The same experiment is conducted in the same manner using MURA bone X-ray data. The results of all the individual split ratios with a different number of end-systems for every seven body parts are represented in Figure 7 . The exact accuracy levels are summarized in Table 2. For all the seven body parts, the split ratio that comprises of a large data portion along with equally split data ratios (i.e., 8:1:1, 7:1:1:1, and 6:1:1:1:1) executes the best performance for the three different end-systems. The results show a clear trend in that the performance is at its peak when the data ratio is split equally with one end-system possessing a majority of the data. In other words, with 5 participating hospitals, the highest accuracy level is achieved when the data is split into a ratio of 6:1:1:1:1, where one hospital has a large portion of the data and the remaining end-systems have an equal amount of data.

Similarly, the effect of varying the split ratio is tested using cholesterol data as well. As it can be observed from the test results in Table 3, the best performances are given when there a group of smaller-sized hospitals joins forces with one big scale hospital. That is to say when the split ratio is distributed so that one end-system holds a large portion of the data and the other end-systems have equally shared the remaining data, the RMSLE loss value is the lowest, hence accomplishing the best outcome. Consequently, the generic pattern monitored across all three different datasets for variations made to the split ratio is summarized as:

- The highest performance in split learning is observed when a hospital holding a large amount of data is paired with other small hospitals that each possess an equal amount of data.

\section{Discussion}

Data sharing can enhance the process of devising treatment plans and diagnosing patients. Furthermore, collecting data from multiple facilities allows scientific breakthroughs to reach another level beyond those obtained from one research. This study evaluates the classification and prediction performance of our multi-site split learning algorithm whilst varying the number of clients and the data ratio.

The results of this study illustrate that medical data can be shared amongst other institutions whilst preserving privacy. From testing with COVID-19 chest CT scans, MURA X-ray images, and cholesterol levels, a correlation between the number of end-systems and the split ratio is formed. As mentioned in the results section, it can be seen that when the data ratio varies from being equally distributed, to having a data imbalance, to extreme data imbalance, the accuracy levels gradually increase. In our experimental setup, when we say that the data is equally shared amongst the participating hospitals, it is analogous to small hospitals with small amounts of data training one neural network. Notice the accuracy levels in Table 1, 2, and 3 is at its peak when the split learning ratio is divided such that one hospital holds a large portion of the data and the other hospitals equally share the remaining data. In other words, in the case where there are 3 end-systems, the best accuracy is seen when the data is divided in a 8:1:1 ratio for all seven of the body portions. The same analysis can be made with the cases of 4 end-systems and 5 end-systems; the highest accuracy level is achieved with a split ratio of 7:1:1:1 and 6:1:1:1:1, respectively.

The reasoning behind this trend becomes more comprehensible when a simple yet logical comparison to a realistic setting is made. When several small private clinics with a lower number of patient records work only with each other to train a deep neural network, it will obviously perform less. However, when an extremely large-scale hospital, such as The Johns Hopkins Hospital or Massachusetts General Hospital joins that group of small clinics in split learning, then more accurate classifications or predictions will be made. Since small local clinics don't accumulate as much data as extensive teaching hospitals, a performance difference is bound to occur. Therefore, having an equal split of data whilst one hospital holds on to the majority of the data, gives the most accurate classification for medical image data. The same pattern is seen across all the datasets tested in this paper.

Theoretically, when the datasets are equally shared among the participants, the problem of internal covariate shift occurs. In other words, since the batch sizes are the same, during training the solution may reach local optimum rather than the global optimum. However, when a large dataset joins the other small groups (i.e., 7:1:1:1), then the distribution of inputs flowing throughout the network shifts to be around the same mean and standard deviation. Thereby, giving a higher performance rate. The results of our study are corroborated both logically and theoretically.

The experiments conducted throughout this study allows us to give empirical verification on the optimal number of end-systems and split ratio. It is finalized that for optimal performance for split learning is to have 5 end-systems with the split ratio being 6:1:1:1:1. To achieve the most efficient classification or prediction performance under split learning, research should be conducted with 5 hospitals where one major hospital with the majority of the data joins four other small clinics that have equal amounts of data among themselves. This conclusion is made based on the consideration of both the split ratios and end-systems. This is a guideline to make harmonized and concerted efforts for those in the medical environment wishing to create more meticulous healthcare developments.

This optimal configuration does not give the highest classification accuracy or lowest loss. The arrangement that gives the best performance is when there are 3 end-systems with a split ratio of 8:1:1. However, we select our proposed composition to 
be the optimal choice because the difference in accuracy levels is only $0.9 \%$. By sacrificing a drop in performance of less than $1 \%$, more hospitals are encouraged to engage in split learning and share their findings to create substantial medical findings. Thereby, forgoing this infinitesimal decline in performance gives smaller hospitals the opportunity to participate in training a common deep neural network.

Our methods allow a transparent use of data, especially in current medical trends where collecting of medical data is on the rise and possessing big data creates paths for scientific advancement. The benefit of split learning in regards to privacy and overcoming data-imbalance can gain consumer trust and willingness to share more information to create meaningful medical deductions. Creating a larger aggregation of datasets will be able to provide a holistic view of emerging health problems. We hope that more partnerships between small local clinics and major teaching hospitals can be formed for fundamental improvements in the medical field to occur.

\section{Methods}

\section{Data Collection or Study Datasets}

We use two different types of data-image and numerical data to give credence to our multi-site split learning algorithm. COVID-19 chest computed tomography (CT) scans and MURA bone X-ray images are used for classification and cholesterol data, a numerical data, is analyzed for prediction. To enhance diagnoses and speed up treatment options for patients in the time of this global epidemic, our original split learning algorithm is used to determine whether the chest CT scan is of a patients suffering from the COVID-19 virus or not. We used chest CT scans of COVID-19 patients obtained from ${ }^{3}$, which is a large compilation of COVID-19 chest CT scans from several public sources ${ }^{4-9}$.

The MURA dataset collected by Stanford University School of Medicine and ML Group ${ }^{10}$ is of X-ray scans of the patient's elbows, fingers, forearms, hands, humerus, shoulders, and wrists. This data is used to promptly diagnose those who suffer from musculoskeletal disorders. If there is a fracture present in the bone X-ray scan, then the patient is classified positive, meaning that he or she or they has this condition. On the other hand, if there is no fracture in the bones, then the patient is classified negative, as in the patient is healthy with their bones intact in one piece.

We also analyzed real-world medical data-cholesterol levels-provided by Seoul National University Hospital (SNUH). This study was approved by the Institutional Review Board of Seoul National University Hospital (No. C-1712-009-903) with a waiver of informed consent. No personally identifiable data was included in the dataset. Data used in this study were retrieved from Seoul National University Hospital's Common Data Model (CDM) database. LDL-C is a type of cholesterol that builds up in the blood vessels and plays part in causing coronary artery disease (CAD) ${ }^{11}$. Hence, detecting the levels of LDL-C in the bloodstream is critical to prevent any health problems. The dataset obtained from SNUH contains the patient's age, sex, height, weight, total cholesterol (TC), high-density lipoprotein cholesterol (HDL-C), triglyceride (TG), and LDL-C. We train a regression model that can use the above attributes to predict the value of LDL-C as accurately as possible.

\section{Split Learning Algorithm}

In this study, we investigate the performance of our multi-site split learning when changes to the number of end-systems and the data distribution are made. All methods were carried out in accordance with relevant guidelines and regulations. For all experiments, the split learning setup is the same, with the deep neural network being divided amongst the end-systems and one centralized server. Even with the varying number of end-systems that are participating in training the deep neural network, each and every end-system only holds one hidden layer. This hidden layer comprises one convolutional layer. The original medical data, be it the COVID-19 CT chest scans or the MURA bone X-ray images, or the numerical cholesterol data, will pass only through the first hidden layer. The output from the first hidden layer is an encrypted feature map. This feature map is distorted to the point where it cannot be used to inference the original data in this multi-site split learning algorithm. This feature map is the only information that is exposed to an external network-the centralized server. The heavily deformed feature map that is transferred to the server is shown in Figure 2 and Figure 3. Since only this encrypted parameter is passed to the server, and not the raw medical images, protection of personal information is ensured.

The feature maps from all the participating hospitals are concatenated and processed as the input to the deep neural network. Since the end-systems only run the neural network up to the first hidden layer, the remaining hidden layers are placed in the server. It should be noted that all the end-systems have the same number of hidden layers-that is, one hidden layer. With the concatenated feature map, the server trains the deep neural network. Considering the concatenated feature map is a summation of all the feature maps from each and every end-system, the deep neural network that is located in the server can learn using all the data from the participating hospitals.

\section{Deep Learning Models}

The deep learning models are selected appropriately to accommodate the data type used. Since the two image datasets-COVID19 CT scans and MURA X-ray scans-require a classification tool, a classification model is used. To determine whether or not a 


\begin{tabular}{l|ccc}
\hline Parameters & Covid-19 chest & MURA & Cholesterol \\
\hline Epochs & 100 & 50 & 200 \\
Loss & Binary crossentropy & Binary crossentropy & MSE \\
Activation function & Sigmoid & Sigmoid & Leaky ReLU \\
Batch size & 64 & 128 & 2,048 \\
Input Size & $64 \times 64 \times 1$ & $224 \times 224 \times 1$ & 326,032 \\
Model & Custom classification & VGG19 & Custom regression \\
\hline
\end{tabular}

Table 4. The arrangements for a deep learning model to train each of the COVID-19, MURA and cholesterol data sets.

certain patient's CT scan is that of a patient suffering from the viral COVID-19 virus, a custom classification model is designed. The epoch is set to 100 , with the binary crossentropy chosen as the loss function. As for the activation function, the sigmoid function is used for this customized model. The input image sizes are all set to a size of $64 \times 64 \times 1$, with a batch size set to 64. This setting is applied to the server where it trains to classify the CT scan from that of a COVID-19 patient to a healthy lung. The end-systems hold one hidden layer while the server trains with 4 hidden layers.

As for the MURA datasets, a deeper VGG19 model is used to train the classification model. Due to the fact that there is a lower number of data compared to the 407,953 images of COVID-19 CT scans, a deeper and more intricate neural network is used. This model is devised with an epoch of 50 and a batch size of 128. The loss and activation functions are binary crossentropy and sigmoid, respectively. The X-ray image sizes are all reshaped to input size of $224 \times 224 \times 1$. The MURA bone X-rays scans are trained with a total of 20 layers: one hidden layer placed in each hospital and the remaining 19 layers placed in the server, where the actual training of VGG19 occurs.

On the other hand, cholesterol data is a numerical dataset. Hence, a prediction model must be used. The deep learning model configuration for each of the three datasets is summarized in Table 4. To accurately predict the level of LDL-C in the bloodstream, we designed a custom regression model with an epoch of 200, loss function of mean squared error (MSE), the activation function of Leaky ReLU, and a batch size of 2,048. The input size for this numerical data represents the number of data used to train the custom regression model. This prediction model has 3 layers, of which one is placed in the contributing hospitals, and the remaining 2 is placed in the server.

A generalization of the deep neural network configuration is shown on the server's side in Figure 1. A hidden layer is a group of a Conv2D layer and MaxPooling2D layer. This framework is a generalization of the deep learning structure that is applied to the three datasets. As it can be concluded, our multi-site split learning algorithm applies to all kinds of data types if the adequate model is selected.

\section{Data availability}

The COVID-19 and MURA data generated or analysed during this study are included in this published article. The cholesterol data is a privately obtained dataset from SNUH is made available from the corresponding author upon reasonable request. Our source codes for the current study are available at https://github.com/annabana17/NatureSplitLearning.

\section{References}

1. Organization, W. H. Who coronavirus (covid-19) dashboard. https://covid19.who.int/.

2. Briggs, A. M. et al. Reducing the global burden of musculoskeletal conditions. Bull. World Heal. Organ. 96, 366 (2018).

3. Zhou, T. et al. The ensemble deep learning model for novel COVID-19 on CT images. Appl. Soft Comput. 98, 106885, DOI: https://doi.org/10.1016/j.asoc.2020.106885 (2021).

4. Zhao, J., Zhang, Y., He, X. \& Xie, P. COVID-CT-dataset: A CT scan dataset about COVID-19. arXiv preprint arXiv:2003.13865 490 (2020).

5. Afshar, P. et al. COVID-CT-MD, COVID-19 computed tomography scan dataset applicable in machine learning and deep learning. Sci. Data 8, 1-8 (2021).

6. Cohen, J. P. et al. COVID-19 image data collection: Prospective predictions are the future. arXiv preprint arXiv:2006.11988 (2020).

7. Morozov, S. et al. Mosmeddata: Chest CT scans with COVID-19 related findings dataset. arXiv preprint arXiv:2005.06465 (2020).

8. Rahimzadeh, M., Attar, A. \& Sakhaei, S. M. A fully automated deep learning-based network for detecting COVID-19 from a new and large lung CT scan dataset. Biomed. Signal Process. Control. 68, 102588 (2021). 
9. Jun, M. et al. COVID-19 CT lung and infection segmentation dataset. Zenodo 20 (2020).

10. Rajpurkar, P. et al. MURA: Large dataset for abnormality detection in musculoskeletal radiographs. arXiv preprint arXiv:1712.06957 (2018).

11. Hirayama, S. \& Miida, T. Small dense ldl: an emerging risk factor for cardiovascular disease. Clin. Chimica Acta 414, 215-224 (2012).

\section{Acknowledgements}

This study was approved by the Institutional Review Board of Seoul National University Hospital (No. C-1712-009-903) with a waiver of informed consent. No personally identifiable data was included in the dataset. Data used in this study were retrieved from Seoul National University Hospital's Common Data Model (CDM) database.

This research is funded by the Ministry of Health and Welfare (HI19C0842).

\section{Author contributions statement}

Y.J.H designed and conducted the experiments, analyzed the data, prepared figures, and wrote the manuscript; G.L. and M.J. aided in the experiments and plotted the graphs; J.K. supervised the research; S.J., S.Y, J.K. and Y.J.H. reviewed and approved the final version of the manuscript.

\section{Competing interests}

The authors declare no competing interests.

\section{Additional information}

To include, in this order: Accession codes (where applicable); Competing interests (mandatory statement).

The corresponding author is responsible for submitting a competing interests statement on behalf of all authors of the paper. This statement must be included in the submitted article file. 